\title{
Bioanalytical strategies in determining immunogenicity
}

\author{
Meenu Wadhwa*,1 \& Robin Thorpe ${ }^{2}$ \\ ${ }^{1}$ National Institute for Biological Standards \& Control, Medicines \& Health Care Products Regulatory Agency, Biotherapeutics \\ Group, Blanche Lane, South Mimms, Potters Bar, Hertfordshire EN6 3QG, UK \\ ${ }^{2}$ Welwyn, Hertfordshire, UK \\ *Author for correspondence: Meenu.Wadhwa@nibsc.org
}

First draft submitted: 16 September 2019; Accepted for publication: 19 September 2019; Published online: 7 November 2019

Keywords: antibody-drug conjugates $\bullet$ assays $\bullet$ assessment $\bullet$ clinical immunogenicity $\bullet$ harmonization $\bullet$ predictive technologies • special issue

For almost two decades now, unwanted immunogenicity continues to be a predominant theme for biological products. This is not surprising in view of the serious consequences that can occur and the fact that assessing immunogenicity is scientifically and technically challenging. While progress with development of methodologies for immunogenicity assessment has been significant, adopting appropriate procedures and strategy is still a demanding exercise.

This Special Focus Issue contains ten articles focused primarily on specific issues relating to determining unwanted immunogenicity of biologicals and cover the current guidance, advances in methodologies, approaches for risk assessment and strategies that can be adopted for mitigating such risk.

The first article by Brumm and Peng [1] is an Editorial in which they discuss the evaluation of in-study cut points to enable appropriate interpretation of clinical immunogenicity results. For this, as per the norm, they recommend the risk-based and multitiered approach for immunogenicity assessment and consider factors important for screening assay cut point factor and confirmatory assay cut point determination. They assess how to best compute cut points and present two case studies as examples to highlight this important issue when implementing cut points (those generated during assay validation using commercial samples vs in-study cut points) for clinical studies.

Shankar, in his Interview [2], considers the current challenges in assessing immunogenicity. These include consideration of predictive technologies, bioanalytical and clinical procedures especially at the regulatory level, and factors relating to assay sensitivity and tolerance. He emphasises that robust methods are needed to ensure that the immunogenicity data is accurate and provides his opinion on the updates in the recently finalized US FDA regulatory guidance. Some perspective on future trends is also included.

Interference in the assay is an important consideration for generating valid data. Pöhler, Faigl and Staack specifically consider issues relating to evaluating potential biotin interference in immunogenicity testing [3]. This includes technical details focused on one-step bridging assays and the steps that can be taken to overcome the problematic issue of biotin interference in sensitive immunoassays.

With four approved antibody-drug conjugates (ADCs) to date and many in clinical development using a variety of ADC platforms, the topic of immunogenicity of ADCs is very timely. Carrasco-Triguer et al. present the strategy used and the results on immunogenicity of eight different ADCs derived using the same platform in 11 clinical trials [4]. Presence of nonhuman epitopes in the conjugates was not found to cause significant clinical problems the incidence of antibody induction in the participants was similar to that described for therapeutic monoclonal antibodies. The authors conclude that while the risk of immunogenicity diminishes with progression through the clinical phases, a conservative approach is recommended for novel ADC platforms using different conjugation chemistries and very potent cytotoxic drugs.

In an article dedicated to assays, Chen $e t$ al. describe ways of mitigating target interference in bridging immunogenicity assays by using particular target-blocking reagents and mild basic $\mathrm{pH}$ [5]. Using such approaches resulted 
in high tolerance to recombinant target protein and reduced levels of positivity in clinical study samples which correlated with expected clinical findings.

In his article, Chamberlain provides practical advice on effective presentation of immunogenicity risk assessments and related data in regulatory dossiers [6]. He focuses on a model for an integrated summary of immunogenicity to be submitted in marketing authorization applications for novel biopharmaceutical products in International Conference on Harmonisation (ICH) regions. The model is well presented and allows assessment of the impact of immunogenicity on overall clinical benefit versus risk.

Thorpe and Wadhwa consider aspects important for the harmonization and standardization of immunogenicity assessment of biotherapeutic products [7]. They provide guidance that hopefully will lead to harmonized practices for immunogenicity assessment and generation of robust immunogenicity data. Standards and reference panels for use in assays employed for immunogenicity assessment are considered as are strategies adopted for immunogenicity studies used for regulatory purposes. In addition, present efforts and future needs for harmonizing clinical monitoring for better patient outcomes are also discussed.

Jiang et al. describe the technical aspects of hybrid ligand-binding assay/LC-MS and explore its use in the clinical immunogenicity setting [8]. They consider their findings to show that the semiquantitative assay developed and described has acceptable specificity, drug tolerance and the capability of achieving multiplexed detection of antibodies induced by biological products. While preliminary data seem promising, the utility of this orthogonal tool in large clinical testing programs remains to be seen.

Kurki assesses the compatibility of immunogenicity guidance provided by the EMA and the FDA [9]. Although these are based on different legislations and regulatory philosophies, they are compatible and harmonized at the scientific level, especially for new innovative therapeutic protein products. The main differences are seen in the approach taken, the detailed and prescriptive FDA guidance versus the flexible and pragmatic view taken in the EU guidance. The technical and regulatory aspects of both guidelines and their implications as well as their view on biosimilars are considered in some detail.

In the final article, Tourdot and Hickling consider the nonclinical immunogenicity risk assessment of therapeutic proteins [10]. They describe methods used to assess the risk of unwanted immunogenicity during preclinical drug development, and discuss approaches to therapeutic protein immunogenicity risk assessment, including assays and in vivo models used to mitigate such risk.

The aim of this Special Focus issue is not to provide a comprehensive evaluation of all aspects of unwanted immunogenicity (this has been adequately provided by several textbooks on the subject), but to highlight some important specific issues worthy of consideration. The success of this will be largely judged by how it influences the assessment of immunogenicity in the future.

Financial \& competing interests disclosure

The authors have no relevant affiliations or financial involvement with any organization or entity with a financial interest in or financial conflict with the subject matter or materials discussed in the manuscript. This includes employment, consultancies, honoraria, stock ownership or options, expert testimony, grants or patents received or pending, or royalties.

No writing assistance was utilized in the production of this manuscript.

\section{References}

1. Brumm J, Peng K. Evaluation of in-study cut points to enable appropriate interpretation of clinical immunogenicity results. Bioanalysis 11(17), 1539-1541 (2019).

2. Shankar G. Current challenges in assessing immunogenicity. Bioanalysis 11(17), 1543-1546 (2019).

3. Pöhler A, Faigle J, Staack RF. Evaluation of potential biotin interference in immunogenicity testing. Bioanalysis 11(17), 1547-1554 (2019).

4. Carrasco-Triguero M, Dere RC, Milojic-Blair M, et al. Immunogenicity of antibody-drug conjugates: observations across 8 molecules in 11 clinical trials. Bioanalysis 11(17), 1555-1568 (2019).

5. Chen J, Garlits J, Dhulipala G, et al. Mitigating target interference in bridging immunogenicity assay with target blocking reagents and mild basic pH. Bioanalysis 11(17), 1569-1580 (2019).

6. Chamberlain P. Effective presentation of immunogenicity risk assessments and related data in regulatory dossiers. Bioanalysis 11(17), 1581-1592 (2019).

7. Wadhwa M, Thorpe R. Harmonization and standardization of immunogenicity assessment of biotherapeutic products. Bioanalysis 11(17), 1593-1604 (2019). 
8. Jiang H, Myler H, Zeng J, Mora J, Kolaitis G, Pillutla R. Perspectives on exploring hybrid LBA/LC-MS approach for clinical immunogenicity testing. Bioanalysis 11(17), 1605-1617 (2019).

9. Kurki P. Compatibility of immunogenicity guidance by the EMA and the FDA. Bioanalysis 11(17), 1619-1629 (2019).

10. STourdot, TP Hickling. Nonclinical immunogenicity risk assessment of therapeutic proteins. Bioanalysis 11(17), 1631-1643 (2019). 